\title{
Behandlung im Voraus planen (Advance Care Planning): ein neues Konzept zur Realisierung wirksamer Patientenverfügungen
}

\author{
Advance Care Planning: A New Concept to Realise Effective Advance \\ Directives
}

\section{Zusammenfassung}

Advance Care Planning - deutsch: Behandlung im Voraus planen (BVP) - etabliert sich inzwischen auch in Deutschland als ein neues Konzept zur Realisierung wirksamer Patientenverfügungen. Dabei erhalten Interessenten zum einen im Rahmen eines professionell begleiteten Gesprächsprozesses Gelegenheit, eigene Präferenzen für künftige medizinische Behandlungen bei Einwilligungsunfähigkeit zu entwickeln und aussagekräftig zu dokumentieren. Zum anderen werden die relevanten regionalen Institutionen und Versorgungsstrukturen in einem systematischen Ansatz (Change Management) beteiligt, sodass die Vorausverfügungen regelmäßig verfügbar sind und zuverlässig beachtet werden.

Der Artikel erläutert zunächst das ethische Fundament von BVP und warum dieses neue Konzept notwendig ist, erklärt das BVP-Grundkonzept und referiert wichtige internationale Entwicklungen in diesem Bereich. Sodann werden die einzelnen Elemente eines BVP-Programms vorgestellt und die Inhalte der Vorausplanungsgespräche erläutert, unter besonderer Berücksichtigung der Vorausplanung für akute medizinische Notfälle und für zum Planungszeitpunkt nicht einwilligungsfähige Personen. Der Beitrag schließt mit Hinweisen zur regionalen Implementierung und zum neuen $\S 132$ g SGB V, der - voraussichtlich ab 2017 - die Implementierung von BVP-Angeboten in stationären Pflegeeinrichtungen und Einrichtungen der Eingliederungshilfe für behinderte Menschen zulasten der GKV vorsieht.

\section{Abstract \\ $\nabla$}

Advance Care Planning (ACP) is now also established in Germany as a new concept to realise effective advance directives. Accordingly, individuals are regularly invited to develop and document their preferences for possible future medical care in a professionally facilitated communication process. At the same time, all relevant health care institutions and lead actors are invited in a systematic regional approach (change management) to participate, to inform and train staff at various levels, and to implement the necessary structures so that the resulting care plans are reliably available and respected.

This paper outlines the ethical foundations of ACP and why this new approach became necessary, explains its basic concept, and reports major international ACP developments. The required elements of an ACP program and the contents of the facilitated communication process are presented in detail. Advance planning for emergency situations is covered as well as planning for individuals who have no decision-making capacity (ACP by proxy). The article closes with directions for the regional implementation of ACP programs, and an introduction into the new $\S 132 \mathrm{~g}$ (Social Code Book V) which effectuates, probably from 2017 on, reimbursement of chronic care institutions that participate in regional ACP programs and offer facilitated ACP to their residents.

Institut für Allgemeinmedizin, Universitätsklinik Düsseldorf

${ }^{2}$ Klinik für Palliativmedizin, Universitätsmedizin Göttingen, Georg-August-Universität Göttingen

${ }^{3}$ Institut für Ethik, Geschichte und Theorie der Medizin, Ludwig-Maximilians-Universität, München
Schlüsselwörter
- Behandlung im Voraus planen
Patientenverfügung
- Patientenautonomie
- Einwilligungsfähigkeit
Keywords
- Advance Care Planning
- patient autonomy
- advance directive
- decision-making capacity
- informed consent

VNR

2760512016149752663

Bibliografie

DoI http://dx.doi.org/

10.1055/s-0042-110711

Z Palliativmed 2016; 17 :

177-195

(c) Georg Thieme Verlag KG

Stuttgart · New York

ISSN 1615-2921

Korrespondenzadresse

Univ.-Prof. Dr. med.

Jürgen in der Schmitten, MPH

Institut für Allgemeinmedizin (ifam),

Universitätsklinik Düsseldorf

Moorenstraße 5

40225 Düsseldorf

jids@med.uni-duesseldorf.de 


\section{Einleitung}

\section{$\nabla$}

Patientenverfügungen gibt es seit Anfang der 1970er-Jahre, und im Prinzip befürworten die meisten Menschen, dass mittels Vorausplanung ungewünschte medizinische Anstrengungen zur Lebensrettung und -verlängerung vermieden werden können. Gleichzeitig sind sich die meisten der Tatsache bewusst, dass Patientenverfügungen seit Jahrzehnten in der realen Welt medizinischer Entscheidungsfindung meist nur eine untergeordnete Rolle spielen und kritische Entscheidungen in Unkenntnis des Patientenwillens an der Tagesordnung sind - zu Hause, im Altenheim oder auf der Intensivstation.

Seit den 1990er-Jahren hat sich mit Advance Care Planning (ACP) - deutsch: Behandlung im Voraus planen (BVP) - eine neue Herangehensweise an Patientenverfügungen entwickelt, die das Potenzial besitzt, diese Defizite erfolgreich zu kompensieren (für eine umfassende deutschsprachige Übersicht s. [1]).

Auch in Deutschland ist das Interesse an diesem Konzept seit dem vergangenen Jahr erheblich gestiegen, nicht zuletzt durch das im Dezember 2015 in Kraft getretene Hospiz- und Palliativgesetz (HPG). Mit dem neuen §132 g SGB V können stationäre Pflegeeinrichtungen und Einrichtungen der Eingliederungshilfe für behinderte Menschen ihren Bewohnern künftig eine „Gesundheitliche Versorgungsplanung für die letzte Lebensphase“ zulasten der Krankenkassen anbieten.

Der vorliegende CME-Beitrag erklärt, was es mit dieser neuen Herangehensweise - Advance Care Planning (ACP) oder Behandlung im Voraus planen (BVP) - auf sich hat, und vermittelt wesentliche Grundkenntnisse zur Implementierung und Durchführung von BVP.

\section{Befähigung als konstitutives Element von Patientenselbstbestimmung \\ $\nabla$}

Bevor darüber gesprochen werden kann, ob Patientenverfügungen wirksam sind oder wie sie künftig wirksamer werden könnten, sollte Einvernehmen über die Prämissen bestehen, die dem Instrument der Patientenverfügung zugrunde liegen.

Die Akzeptanz vorausverfügter Behandlungswünsche gründet - ebenso wie die Akzeptanz aktueller Behandlungsentscheidungen von Patienten überhaupt - auf der Annahme, dass von allen infrage kommenden Personen der Betroffene letztlich am besten selbst weiß, was zu seinem Wohle ist - nicht die Angehörigen oder der Betreuer, nicht der Arzt, nicht die Bezugspflegekraft und auch sonst niemand anderes.

Entscheidungen, die das Wohl des Betroffenen („salus aegroti“) im Blick haben, befinden sich regelmäßig in einem Spannungsfeld zwischen den ethischen Verpflichtungen des Wohltuns („beneficence“) und des Nichtschadens („nonmaleficence“) [2]. Entscheidend für die Bewertung, ob der Versuch einer Maßnahme genügend Erfolg verspricht und das damit meist verbundene Schadensrisiko rechtfertigt, sind die vorausgehende Festlegung des individuellen Behandlungsziels sowie davon abhängig die Gewichtung der Chancen, Belastungen und Risiken.

Bei näherem Hinsehen wird deutlich, dass dies im Kern Wertentscheidungen erfordert, die sich nicht aus dem medizinischen Fachwissen ergeben. Diese Wertentscheidungen ohne Wenn und Aber dem entscheidungsfähigen Patienten zuzugestehen, auch wenn dessen Präferenzen aus fachlicher Sicht manchmal irritieren mögen, ist Ausdruck des Prinzips der Selbstbestimmung (Autonomie), das wesentlich durch die Rechtsprechung in einem über 100-jährigen Prozess entwickelt und anstelle des bis weit ins 20. Jahrhundert hinein verbreiteten Paternalismus etabliert worden ist.

Die Rechtsprechung hat aber auch herausgearbeitet, dass Patientenselbstbestimmung an bestimmte Voraussetzungen gebunden ist, damit sie überhaupt wirksam ausgeübt werden kann. Wie auch anderswo im Rechtswesen ist eine wirksame Entscheidung durch den Patienten nur möglich, wenn dieser alle entscheidungsrelevanten Informationen erhalten, verstanden und gewürdigt hat. In der Medizin heißt dieses Prinzip Einwilligung nach Aufklärung (oder Informed Consent) [3].

So nüchtern das klingt, „alle entscheidungsrelevanten Informationen ... gewürdigt hat“, sosehr zeigt ein Blick in unsere Lebenswelt, dass in der Regel - besonders bei schwierigen und folgenreichen Entscheidungen - ein komplexer Austausch mit anderen Personen erforderlich ist, bis wir uns in der Lage fühlen, unsere eigene, höchst individuelle Entscheidung zu treffen - unter Berücksichtigung dessen (aber nicht dirigiert von dem), was andere, die uns wichtig sind, uns dazu gesagt oder auch entgegengehalten haben. Das Verständnis von Selbstbestimmung, das einen sozialen Prozess des Miteinanders als Voraussetzung für fundierte Entscheidungen ansieht, die wir nach bestem Wissen und Gewissen verantwortlich treffen, kann als relationale Autonomie bezeichnet werden [4]. 


\section{Voraussetzungen}

Patientenverfügungen erfüllen nur dann ihren Zweck - effektive Wahrung der Selbstbestimmung bei Nichteinwilligungsfähigkeit -, wenn sie

1. in ausreichender Zahl erstellt wurden (Prävalenz),

2. aussagekräftig für relevante klinische Szenarien formuliert wurden (Aussagekraft),

3. verlässlich den wohlinformierten Willen der Betroffen wiedergeben (Validität),

4. in der Entscheidungssituation tatsächlich vorliegen (Verfügbarkeit) und schließlich

5. im Anwendungsfall angemessen von Ärzten und anderem Gesundheitspersonal beachtet werden (Beachtung).

In der Medizin sind wichtige Menschen, meist Angehörige oder gute Freunde, mit denen zentrale Entscheidungen des Lebens sonst gern besprochen werden, häufig nicht zugegen, wenn kritische Behandlungsentscheidungen getroffen werden müssen, oder sie eignen sich nicht als Gesprächspartner. Zudem ist es der behandelnde Arzt, der die medizinische Situation und die gegebenen Handlungsoptionen am besten kennt und häufig gleichzeitig über eine tragfähige Beziehung zum (idealerweise seit längerer Zeit betreuten) Patienten verfügt.

Damit selbstbestimmte Behandlungsentscheidungen möglichst valide, nämlich im Sinne einer relationalen Autonomie, getroffen werden können, bedarf es daher einer Arzt-Patient-Beziehung, die einerseits getragen ist von einer Haltung des Respekts, dass der Patient letztlich die richtige Entscheidung für sich treffen wird, und sei sie aus ärztlicher Sicht oder nach ärztlicher Erfahrung auch noch so fernliegend, und die andererseits ein partnerschaftliches, authentisches, empathisches und durchaus auch kritisches Miteinander pflegt, das zudem durch Informationsflüsse in beide Richtungen geprägt ist. Damit entsteht ein Beziehungsraum, innerhalb dessen der Patient seine Präferenzen häufig überhaupt erst entwickeln, kritisch prüfen und schließlich festlegen kann. Diese gemeinsame Entscheidungsfindung (Shared Decision Making) stellt die Grundlage dar, auf der wirksame Einwilligungen gemäß dem Prinzip des Informed Consent gedeihen können [5].

Das heißt aber auch: So verstandene Selbstbestimmung realisiert sich nicht einfach durch eine bloße Meinungs- oder Willensäußerung seitens des Patienten - ganz besonders in einem Bereich, der so von Asymmetrie (hinsichtlich Informationen und Machtgefüge) gekennzeichnet ist wie die Medizin. Soll der Patient vielmehr wirksam in eine Maßnahme einwilligen oder sie ablehnen können, so muss er dazu befähigt werden durch einen höchst anspruchsvollen Beziehungs- und kommunikativen Prozess [6]. Diese Voraussetzungen, so wird im Folgenden deutlich, müssen auch bei der Vorausplanung von möglichen zukünftigen Behandlungsentscheidungen erfüllt werden.

\section{Ungelöste Probleme konventioneller Patientenverfügungen}

Bei Verlust der Einwilligungsfähigkeit durch eine schwere Erkrankung kann die Selbstbestimmung der betroffenen Patienten nur dann berücksichtigt werden, wenn die Behandlungswünsche - nach entsprechender Aufklärung - vorab ermittelt und dokumentiert wurden. Im Jahr 2009 wurde mit dem 3. Gesetz zur Änderung des Betreuungsrechts (sog. Patientenverfügungsgesetz) auch gesetzlich verankert, dass die in einer Patientenverfügung schriftlich dokumentierten Behandlungswünsche zu beachten sind, insoweit sie auf die gegebene Situation zutreffen. Verschiedene empirische Untersuchungen belegen, dass diese Voraussetzungen in Deutschland nicht flächendeckend und regelhaft erfüllt sind: Patientenverfügungen sind

nach wie vor zu wenig verbreitet,

- häufig nicht aussagekräftig formuliert,

v von fragwürdiger Validität,

bei Bedarf oft nicht zur Hand und

- bleiben vom medizinischen Personal nicht selten unbeachtet [7-9].

Angesichts vergleichbarer internationaler Erfahrungen wurde das Instrument der Patientenverfügung bereits für gescheitert erklärt [10]. Bei näherer Betrachtung vermag diese Diagnose allerdings nicht zu überzeugen, im Gegenteil: Eine effektive Vorausplanung von Behandlungsentscheidungen ist nicht nur ethisch geboten, sondern auch möglich.

Erforderlich ist aber ein neuer konzeptioneller Ansatz, bei dem die Verantwortung für die Erstellung und Umsetzung von Patientenverfügungen und Vorsorgevollmachten nicht mehr dem Einzelnen allein überlassen bleibt, sondern von den Institutionen bzw. Verantwortlichen des regio-

- Trotz gesetzlicher Verankerung der Patientenverfügung sind die Voraussetzungen für eine effektive Berücksichtigung der Selbstbestimmung bei Verlust der Einwilligungsfähigkeit nicht flächendeckend und regelhaft erfüllt.
- Eine effektive Vorausplanung von Behandlungsentscheidungen ist ethisch geboten und mittels Advance Care Planning auch möglich. 
BVP bewahrt Patienten vor ungewollter Über- oder Untertherapie und kann zudem Angehörige, Bevollmächtigte/Betreuer sowie Pflegende und Ärzte bei den stellvertretenden Entscheidungen entlasten.

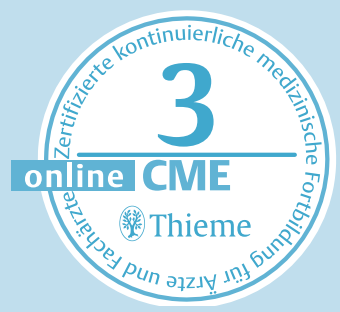

nalen Versorgungssystems übernommen wird. Solch ein systemischer Ansatz, der den Einzelnen bei der Vorausplanung unterstützt - im Sinne einer gemeinsamen Entscheidungsfindung und die regelhafte Umsetzung der Behandlungswünsche sicherstellt, wurde mit Advance Care Planning zunächst in den USA entwickelt und zunehmend international sowie auch in Deutschland erfolgreich etabliert (s. u.).

\section{Ein neues Konzept: Behandlung im Voraus planen (Advance Care Planning) $\nabla$}

\section{Begriffsbestimmung}

Bislang konnte sich noch keine Übertragung von „Advance Care Planning“ ins Deutsche allgemein durchsetzen. Verwendet wurden u. a. Begriffe wie

- "gesundheitliche Vorausplanung“,

- „vorausschauende Versorgungsplanung“ und

- „vorausschauende Behandlungsplanung“.

Der neue §132g SGB V spricht von einer „gesundheitlichen Versorgungsplanung für die letzte Lebensphase“. Die Autoren schlagen vor, für das Konzept Advance Care Planning die Bezeichnung „Behandlung im Voraus planen“ - kurz: BVP - zu verwenden, da diese in leicht verständlicher, griffiger Form den Kern des ACP-Prozesses verdeutlicht.

\section{Prinzip}

Es geht bei Advance Care Planning vor allem darum, Behandlungen für mögliche zukünftige medizinische Entscheidungen im Voraus zu planen.

\section{Definition von Behandlung im Voraus planen (BVP)}

Behandlung im Voraus planen (BVP) verfolgt das Ziel, mögliche künftige medizinische Entscheidungen so vorauszuplanen, dass Patienten auch dann zuverlässig nach ihren individuellen Wertvorstellungen und Wünschen behandelt werden, wenn sie diese krankheitsbedingt nicht mehr selbst äußern können. Zudem können auch Wünsche und Präferenzen zu anderen künftig relevanten Aspekten geäußert und festgelegt werden, z.B. pflegerischer, psychosozialer oder spiritueller Art. Nicht zuletzt können auch medizinische Indikationen für das Vorgehen im Notfall im Voraus überprüft und ein Handeln gemäß den Regeln ärztlicher Kunst - z. B. im Fall eines Herzstillstands - rechtzeitig gebahnt werden.

BVP bewahrt nicht einwilligungsfähige Betroffene vor ungewollter Über- oder Unterversorgung und hat darüber hinaus das Potenzial, Angehörige bzw. Bevollmächtigte/Betreuer sowie Pflegende und Ärzte, die stellvertretend weitreichende Entscheidungen treffen bzw. mit deren Folgen leben müssen, vor vermeidbaren psychischen Belastungen zu schützen.

Diese Zielsetzung erreicht BVP durch Interventionen auf 2 Ebenen (s. Infobox „Prinzip“).

\section{Prinzip}

\section{Grundelemente von BVP}

- Auf der individuellen Ebene erhalten die Mitglieder der Zielgruppe im Rahmen eines qualifizierten, professionell begleiteten Gesprächsprozesses - im Sinne einer vorweggenommenen gemeinsamen Entscheidungsfindung (Shared Decision Making) - Gelegenheit, eigene Präferenzen für künftige medizinische Behandlung bei Verlust der Einwilligungsfähigkeit zu entwickeln und in aussagekräftigen, (zumindest) regional einheitlichen Vorausverfügungen zu dokumentieren.

- Auf der Systemebene werden alle relevanten regionalen Institutionen und Versorgungsstrukturen eingebunden und die dort tätigen Personen so geschult bzw. informiert, dass die resultierenden Vorausverfügungen im Fall zu treffender Behandlungsentscheidung regelmäßig verfügbar sind und zuverlässig respektiert werden. 


\section{Historische Entwicklung und aktueller Stand der Implementierung}

\section{Internationale Entwicklungen}

„Respecting Choices“ (La Crosse, USA)

Die Entwicklung eines der ersten umfassenden Advance Care Planning-(ACP-)Programme weltweit begann vor rund 25 Jahren in der Region La Crosse im US-Bundesstaat Wisconsin [11]. Das ACP-Programm „Respecting Choices“ (RC) ist dort inzwischen fest in der regionalen Gesundheitsversorgung etabliert und spielt sowohl in den USA als auch international eine Vorreiterrolle bei der Implementierung von regionalen bzw. nationalen BVP-Programmen.

Zentrale Bausteine dieser Implementierung sind

- ein systematischer Ansatz der Vorausplanung,

- eine standardisierte, kompetenzbasierte Ausbildung von BVP-Gesprächsbegleitern,

- eine Einbeziehung und Aufklärung der Bevölkerung und

- eine kontinuierliche Qualitätssicherung.

Empirische Studien belegen die Effektivität des Programms RC: Im Jahr 2008 konnte in 96\% der Sterbefälle auf eine Patientenverfügung oder eine ärztliche Anordnung über lebensverlängernde Maßnahmen zurückgegriffen werden, bei 93\% der Versterbenden war die Frage einer Herz-Lungen-Wiederbelebung geklärt, die Frage der Krankenhauseinweisung bei 65\% [12]. Die durchgeführten Behandlungen stimmten in 99,5\% der Fälle mit den vorausverfügten Festlegungen überein.

Diese bemerkenswerten Ergebnisse sind das Ergebnis konstanter, mit ausreichenden personellen und finanziellen Ressourcen unterlegter Bemühungen um die Aufrechterhaltung, Entwicklung und Verbesserung des ACP-Programms RC in der Region La Crosse.

\section{„Respecting Patient Choices“ (Australien)}

Eine der erfolgreichsten internationalen Adaptionen von RC stellt das Programm „Respecting Patient Choices" in Australien dar. In verschiedenen Projekten wurde dort seit 2002 unter Förderungen von Bund und Ländern ein landesweites BVP-System etabliert (advancecareplanning. org.au) [13].

An der Universitätsklinik in Melbourne wurde das Programm im Rahmen einer prospektiven, randomisierten und kontrollierten Studie evaluiert [14]. Positive Effekte zeigten sich in der BVP-Interventionsgruppe hinsichtlich der Bekanntheit und Beachtung individueller Behandlungswünsche und der Zufriedenheit mit dem Krankenhausaufenthalt; zudem wiesen die Angehörigen Verstorbener signifikant weniger Symptome von Depression und posttraumatischer Belastungsstörung auf und äußerten eine höhere Zufriedenheit mit dem miterlebten Sterbeprozess.

\section{"Our Voice“ (Neuseeland)}

In Neuseeland wurden seit 2010 von einer Nationalen ACP-Kooperative ebenfalls bemerkenswerte Anstrengungen unternommen, allen Einwohnern des Landes Zugang zu einer effektiven Vorausplanung medizinischer Behandlungsentscheidungen zu ermöglichen (www.advancecare planning.org.nz). Ein Schwerpunkt der neuseeländischen Strategie „Our Voice“ liegt auf einer vierstufigen BVP-Qualifizierung von Mitarbeitern im Gesundheitswesen sowie auf der Förderung eines öffentlichen Bewusstseins für das Thema Vorausplanung, u.a. durch nationale Aktionstage und die Befähigung ehrenamtlicher Helfer [15].

\section{Sonstige BVP-Programme}

Weitere interessante BVP-Implementierungen - jeweils in unterschiedlichem Ausmaß und mit unterschiedlichen Schwerpunkten - in Ländern wie z.B. Kanada, Großbritannien oder Singapur können hier aus Platzgründen nicht dargestellt werden.

\section{Entwicklungen im deutschsprachigen Raum}

Das erste den Autoren bekannte deutsche BVP-Projekt ist das Münsteraner LIMITS [16], das jedoch keine weitere Verbreitung fand.

beizeiten begleiten: Im Rahmen eines BMBF-geförderten Forschungsprojekts (2009-2011) wurde in enger Anlehnung an internationale BVP-Standards und speziell an das Programm Respecting Choices das regionale BVP-Programm beizeiten begleiten entwickelt [17]. In einer interregional kontrollierten Studie konnte gezeigt werden, dass das Programm geeignet ist, nicht nur die Anzahl, sondern auch die Qualität der Patientenverfügungen (insbesondere Aussagekraft und Validität) im Beobachtungszeitraum signifikant zu steigern [18]. beizeiten begleiten wurde anschließend - ohne externe finanzielle Förderung - von 2 der Interventionseinrichtungen in die Regelversorgung übernommen, eine Einrichtung praktiziert es bis heute. 
- Allen Mitgliedern der jeweiligen Zielgruppe wird eine qualifizierte Gesprächsbegleitung zur Erstellung einer Vorausverfügung angeboten.

$\checkmark$ spe Spezifisch geschulte Gesprächs begleiter unterstützen die Betroffenen bei der Entwicklung und Dokumentation ihrer Präferenzen für mögliche künftige Behandlungen.

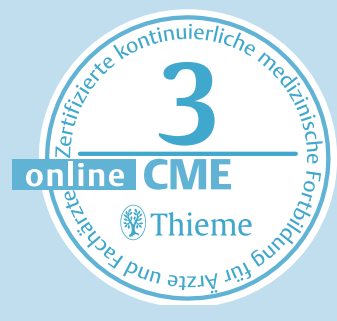

Eine zunehmende Anzahl weiterer Institutionen, Träger und Netzwerke aus ganz Deutschland hat seit dem Jahr 2015 - durchaus mit Blick auf den neuen §132 g SGB V - begonnen, beizeiten begleiten zu implementieren bzw. entsprechende Vorbereitungen zu treffen.

\section{BVP in der Schweiz und in Österreich}

Die MAPS-Studie am Universitätsspital Zürich verbindet eine patientenorientierte Gesprächsbegleitung mit evidenzbasierten Entscheidungshilfen als Grundlage für einen gemeinsamen Entscheidungsprozess hinsichtlich möglicher zukünftiger Behandlungsentscheidungen bei Verlust der Einwilligungsfähigkeit. Die Effektivität der Intervention wird in einer kontrollierten Studie mit schwerkranken Patienten mit einer erwarteten begrenzten Lebenserwartung untersucht [19]. Auch in Österreich gibt es Bestrebungen, BVP zu implementieren [20].

\section{Elemente eines BVP-Programms \\ $\nabla$}

\section{Aufsuchendes Gesprächsangebot}

Bislang bleibt die Initiative zur Abfassung einer Patientenverfügung dem Betroffenen selbst überlassen, wodurch nur ein kleiner Anteil der Bevölkerung eine Vorausverfügung erstellt hat. In einem BVP-Programm übernehmen die regionalen Akteure im Gesundheitswesen die Verantwortung, auf die jeweilige Zielgruppe (z.B. die Bewohner von Senioreneinrichtungen oder alle Bürger ab dem 60. Lebensjahr) zuzugehen, ihnen die Möglichkeit und Vorteile einer Vorausplanung von Behandlungsentscheidungen zu erläutern und ihnen unverbindlich die Unterstützung durch eine professionelle Gesprächsbegleitung anzubieten. Mit diesem aufsuchenden Gesprächsangebot kann die Prävalenz von Vorausplanungen in der Zielgruppe deutlich gesteigert werden [18].

\section{Qualifizierte Gesprächsbegleitung}

Das zentrale Element eines BVP-Programms ist ein qualifizierter Gesprächsprozess, der die Betroffenen bei der Entwicklung und Dokumentation ihrer Präferenzen für mögliche künftige Behandlungen unterstützt.

Spezifisch hierfür geschultes nichtärztliches und ärztliches Personal begleitet den meist mehrzeitigen Gesprächsprozess, der in der Regel in eine individuelle schriftliche Vorausverfügung mündet. Um den nichtdirektiven Charakter der Unterstützung zu verdeutlichen, wird die neue, in der Regel von nichtärztlichem Personal übernommene professionelle Rolle im Englischen als Facilitator bezeichnet, wir sprechen von BVP-Gesprächsbegleiter.

\section{Praxis}

Sofern möglich und gewünscht, sollten die designierten Vertreter (Bevollmächtigte) und/ oder Angehörigen an den Gesprächen beteiligt werden.

Die qualifizierte Gesprächsbegleitung soll im Rahmen eines gemeinsamen Entscheidungsprozesses (Shared Decision Making) einen Informed-Consent-Standard für die vorausverfügten Entscheidungen erreichen und damit aussagekräftige und valide (verlässliche) Vorausplanungen für zukünftige medizinische Entscheidungen ermöglichen. Die erforderlichen Inhalte des Vorausplanungsgesprächs werden unten näher erläutert.

\section{Professionelle Dokumentation}

Regional einheitliche, aussagekräftige Formulare sollen sicherstellen, dass die im Gesprächsprozess ermittelten Präferenzen so dokumentiert werden, dass sie von allen Akteuren im Gesundheitswesen effektiv umgesetzt werden können. Sie sollten dabei die inhaltliche Struktur des begleiteten Gesprächsprozesses abbilden (s.u.). Insbesondere bei älteren oder chronisch kranken Personen sollten in einem separaten Notfall- oder Krisenbogen Behandlungswünsche für den Fall einer plötzlichen gesundheitlichen Krise mit Verlust der Entscheidungsfähigkeit festgehalten werden (s.u.).

Auch bei (dauerhaft) nicht mehr einwilligungsfähigen Personen sollte eine Vorausplanung vorgenommen und in einer Vertreterverfügung dokumentiert werden (s. u.). Neben dem Betroffenen (bzw. dem gesetzlichen Vertreter) unterzeichnen der Gesprächsbegleiter und der ebenfalls spezifisch fortgebildete und beteiligte (Haus-)Arzt das Formular des Notfall- oder Krisenbogens, um damit den qualifizierten Gesprächsprozess zu dokumentieren und die Validität der Vorausplanung für Dritte nachvollziehbar zu machen. 


\section{Archivierung, Zugriff und Transfer}

Im regionalen Versorgungssystem stellen entsprechende Standards und Routinen sicher, dass die entstandenen Vorausverfügungen im Bedarfsfall auch tatsächlich verfügbar sind. Dies umfasst u.a.:

\section{Wichtig}

\section{Archivierung, Zugriff, Transfer}

Die Verfügung ist in den Patienten- bzw. Bewohnerakten an prominenter, leicht auffindbarer Stelle abzulegen. Bei Verlegung des Bewohners einer Pflegeeinrichtung in ein Krankenhaus ist eine Kopie der Verfügung stets Teil der Überleitung.

\section{Aktualisierung und Konkretisierung im Verlauf}

Die schriftliche Vorausverfügung wird von allen Beteiligten nur als Zwischenergebnis eines kontinuierlichen, lebenslangen Gesprächsprozesses angesehen. Eine Wiederaufnahme der Gesprächsbegleitung ist sinnvoll insbesondere

- bei neu auftretenden oder sich verschlechternden chronischen Erkrankungen,

- nach überstandenen stationären Aufenthalten oder

- bei Verlust nahestehender Menschen.

Falls es nicht zu solchen Ereignissen kommt, sollte die Vorausverfügung in regelmäßigen Abständen z.B. im Rahmen von hausärztlichen Vorsorgeuntersuchungen angesprochen und ggf. aktualisiert werden.

\section{Beachtung und Befolgung durch Dritte}

Alle an der Patientenbetreuung Beteiligten in der Region werden darin geschult, die so entstandenen Vorausverfügungen zu beachten und im Bedarfsfall angemessen umzusetzen. Bei der Neuimplementierung eines BVP-Programms sind hierfür entsprechende Informationsveranstaltungen für alle beteiligten Berufsgruppen und Institutionen und die detaillierte Verankerung von BVP in den einschlägigen institutionellen Routinen (z. B. des Qualitätsmanagements) erforderlich. Insbesondere die Befolgung von Notfall- und Krisenbögen durch den Rettungsdienst erfordert eine gründliche Vorbereitung aller Beteiligten.

\section{Kontinuierliche Qualitätssicherung}

Jedes BVP-Programm sollte regelmäßig mit geeigneten Maßnahmen die Prozess- und Ergebnisqualität überprüfen und ggf. verbessern, um eine verlässliche Vorausplanung zu gewährleisten. Von besonderer Bedeutung - und gleichzeitig die größte Herausforderung - ist dabei die Evaluierung der Qualität der Gesprächsbegleitung.

\section{Inhalte der Vorausplanung}

Es ist auffällig, dass in der Vergangenheit vielfach wenig darauf geachtet wurde, welche Inhalte oder Szenarien eine Patientenverfügung abdecken sollte, um für den Großteil der klinisch relevanten Situationen im Sinne des Patientenverfügungsgesetzes ( $§ 1901$ a Abs. 1 BGB) Orientierung zu bieten. In vielen Verfügungen anzutreffende Beschränkungen lebensverlängernder Behandlungsoptionen für den Fall, dass man „irreversibel im Sterben liege“, regeln gar nichts, sie sind ein „Autonomie-Placebo“, da in einer derart beschriebenen Situation lebensverlängernde Maßnahmen ohnehin medizinisch strikt kontraindiziert wären.

So klärten in einer Studie nur 11 von 98 (11\%) Vorausverfügungen aus 10 Senioreneinrichtungen die Frage eines Wiederbelebungsversuchs bei Herzstillstand, obwohl dies aus Sicht der Betroffenen wie auch aus der des Pflegepersonals besonders dringlich ist [18]. Der gelegentlich zu hörende Einwand, man könne ohnehin nicht „alle“ relevanten Szenarien vorab besprechen, greift nicht, da eine genaue Betrachtung zeigt, dass zahlreiche unterschiedliche Einzelverläufe letztlich auf wenige gemeinsame Endstrecken zusteuern, für die sich durchaus effektiv planen lässt - im Folgenden werden die einzelnen Gesprächsabschnitte im Detail beschrieben. BVP verfolgt den Anspruch, diese relevanten Themen und Szenarien sowohl gemeinsam zu besprechen als auch das Besprochene so zu dokumentieren, dass es für die zu treffenden Entscheidungen handlungsleitend wird.

Bei BVP liegt der Fokus auf dem Gesprächsprozess, nicht auf dem schriftlichen Dokument, das nur Ausdruck eines solchen Prozesses sein soll. Als solchem kommt freilich auch der schriftlichen Dokumentation erhebliche Bedeutung zu.

Gegenstand der Vorausplanung sind exemplarische Verläufe, die sich bei unterschiedlichen Erkrankungen oder Verletzungen - als gemeinsame Endstrecke - ergeben können. 
Internationale und eigene Erfahrungen lehren, dass BVP-Gespräche 3 Teile umfassen sollten [17]:

\section{Übersicht}

\section{Inhalte eines BVP-Gesprächs}

- Zunächst sollte im freien Gespräch die Einstellung des Betroffenen zum (Weiter-)Leben und generell zum Einsatz medizinischer Maßnahmen bei schwerer Erkrankung ermittelt werden - im Sinne einer Klärung des Lebenswillens generell sowie bedingter Behandlungsziele.

Diese Ausführungen dienen zum einen als Grundlage für die dann folgende Planung konkreter medizinischer Entscheidungssituationen, zum anderen im späteren Anwendungsfall als Orientierung bei medizinischen Situationen, die nicht explizit von der Vorausverfügung abgedeckt sind.

- Sodann sollten Behandlungspräferenzen für mögliche zukünftige medizinische Entscheidungssituationen geklärt und dokumentiert werden, für die sinnvollerweise die gewünschten Behandlungsmaßnahmen vorausgeplant werden können und sollten. Es erscheint dabei sinnvoll, 3 Szenarien zu besprechen, die sich durch die jeweils verfügbare Information über die Prognose unterscheiden:

- akute medizinische Krisen mit plötzlichem Verlust der Einwilligungsfähigkeit (es ist keine prognostische Information verfügbar),

akute schwere Erkrankung mit anhaltender Nichteinwilligungsfähigkeit (ein Spektrum unterschiedlicher Verläufe und Ergebnisse ist möglich) und

chronische Erkrankungen mit anhaltender Nichteinwilligungsfähigkeit (eine dauerhafte kognitive Einschränkung ist sicher).

- Schließlich sollte eine Vertrauensperson ermittelt und benannt werden, die als Stellvertreter für zukünftige medizinische Entscheidungen bevollmächtigt wird.

Die einzelnen thematischen Elemente der Vorausplanung werden im Folgenden näher erläutert. Darüber hinaus können Wünsche für die zukünftige Pflege sowie für künftige psychosoziale und spirituelle Bedürfnisse erörtert und dokumentiert werden.

\section{Allgemeine Einstellungen zum Leben, Klärung des Behandlungsziels}

In diesem ersten Gesprächsabschnitt gilt es zu ermitteln, wie viel das (Weiter-)Leben dem Betroffenen bedeutet, welches übergeordnete Ziel die medizinische Behandlung verfolgen soll und welche Belastungen und Risiken er - wenn überhaupt - bereit ist, zur Erhaltung seines Lebens auf sich zu nehmen. Diese Grundorientierung hilft im weiteren Verlauf des Gesprächs bei der Einordnung von Antworten zu konkreten medizinischen Maßnahmen oder Szenarien (Kongruenzprüfung). Es kann nicht genug hervorgehoben werden, dass dieser Gesprächsabschnitt ganz entscheidend ist für die spätere Validität der Patientenverfügung und hohe Anforderungen an die kommunikative Kompetenz des Gesprächsbegleiters stellt.

Zu den hierfür wesentlichen Gesprächstechniken zählen

> der thematisch fokussierte narrative Stimulus („erzählen Sie mal“) und

- die vertiefende Exploration („Sie fänden die Vorstellung also schrecklich, nicht mehr für sich selbst sorgen zu können. Was meinen Sie damit?“).

Als hilfreiche, thematisch fokussierende Fragen haben sich hierfür u.a. bewährt:

\section{Übersicht}

\section{Fragen zur Klärung des prinzipiellen Therapieziels}

- Was bedeutet es für Sie, noch (lange weiter) zu leben?

Was hält Sie am Leben, was macht Ihnen Freude?

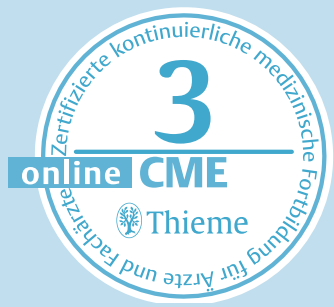

- Können Sie sich einen Krankheitsverlauf/Krankheitsfolgen vorstellen, bei dem/denen Sie sagen würden: Wenn das einträte, wäre mein Lebenswille stark vermindert oder nicht mehr gegeben?

- Wenn Sie an das Sterben denken: Was kommt Ihnen dann in den Sinn?

> Wenn Sie jetzt erfahren würden, dass Sie heute Nacht friedlich einschlafen und morgen nicht mehr aufwachen würden - was würde das für Sie bedeuten? 
Zudem ist die Bereitschaft zu ermitteln, im Krankheitsfall medizinische Hilfe zur Lebensverlängerung anzunehmen. Dabei ist zu klären, welche Risiken (z. B. für unerwünschte Verläufe, etwa schwerwiegende Einschränkungen der Teilhabefähigkeit) und Belastungen der Betroffene dafür in Kauf zu nehmen bereit ist.

Hilfreiche Fragen (die Äußerung eines grundsätzlichen Lebenswillens im vorausgehenden Gespräch vorausgesetzt!) sind in der Infobox zusammengefasst:

\section{Übersicht}

\section{Fragen zur Akzeptanz von Belastungen und Risiken medizinischer Maßnahmen}

- Was soll, was darf Medizin dazu beitragen, Ihr Leben zu verlängern?

- Gibt es Belastungen oder Risiken, angesichts derer Sie keine lebensverlängernden Behandlungen mehr wünschen würden?

> Was müsste gewährleistet sein, damit Sie mit einer lebensverlängernden Behandlung durch die medizinische Behandlung einverstanden wären?

- Welche Sorgen oder Ängste bewegen Sie, wenn Sie an künftige medizinische Behandlung denken? Was soll auf keinen Fall geschehen?

Darüber hinaus kann man die Betroffenen einladen, von persönlichen Erfahrungen (bei sich selbst oder bei anderen Menschen) zu berichten, die die Einstellung zur Frage einer Lebensverlängerung durch medizinische Behandlung bei Verlust der Entscheidungsfähigkeit geprägt haben.

\section{Akute medizinische Krise mit plötzlichem Verlust der Einwilligungsfähigkeit}

Ausführliche Patientenverfügungen sind in akuten medizinischen Notfallsituationen in der Regel kaum anwendbar. BVP-Programme schließen diese Lücke mittels eines Notfall- oder Krisenbogens, der auf nur einer Seite klare Vorgaben für das Rettungspersonal enthält, bis zu welcher Grenze lebensverlängernde Behandlungsmaßnahmen erlaubt sind. Diese Vorausplanung für Notfall- und Krisensituationen hat insbesondere bei älteren oder chronisch kranken Menschen eine hohe praktische Relevanz, da diese das medizinisch Mach- und Vertretbare erfahrungsgemäß häufig und zum Teil erheblich begrenzen wollen.

Der für beizeiten begleiten entwickelte Notfallbogen, die (Haus-)Ärztliche Anordnung für den Notfall (HAnNo), wird weiter unten ausführlich beschrieben.

\section{Akute schwere Erkrankung mit anhaltender Nichteinwilligungsfähigkeit}

Anschließend sollte mit den Betroffenen besprochen werden, wie sie im Fall einer akuten schweren Erkrankung mit voraussichtlich länger anhaltender Nichteinwilligungsfähigkeit behandelt werden möchten. Als exemplarische Fälle sollten hierfür erörtert werden:

- eine schwer verlaufende Pneumonie, die eine intensivmedizinische Behandlung mit längerer Beatmung erforderlich macht, sowie

- ein schwerer Schlaganfall mit ausgeprägter Bewegungseinschränkung sowie Schwierigkeiten zu schlucken, zu sprechen und sich mitzuteilen, in Verbindung mit der Unfähigkeit, selbst zu entscheiden.

In solchen Situationen mit ungewissem Ausgang sind günstigere und ungünstigere Verläufe vorstellbar. Im konkreten Erkrankungsfall besteht die Möglichkeit, ärztlicherseits anhand der gesammelten klinischen Daten eine Prognose abzugeben, und zwar nicht nur bezüglich des Überlebens (quoad vitam), sondern auch hinsichtlich des funktionellen Ergebnisses und daraus folgend der künftig wieder möglichen sozialen Teilhabe.

Im Begleitungsgespräch können Anhaltspunkte dafür erarbeitet werden, welche Belastungen und Risiken für den Betreffenden akzeptabel wären und welche nicht, sodass eine Art prognostischer Behandlungskorridor entsteht, der durch für den Betroffenen individuell nicht akzeptable prognostische Konstellationen (oder aber rein durch das medizinisch Vertretbare) begrenzt wird.

\section{Chronische Erkrankungen mit dauerhafter Nichteinwilligungsfähigkeit}

Des Weiteren sind Behandlungswünsche für den Fall gesundheitlicher Komplikationen bei einem dauerhaften Verlust der Einwilligungsfähigkeit zu besprechen, etwa infolge progredienter Demenz unterschiedlicher Genese oder einer schweren Hirnblutung.

In diesem Szenario ist die Prognose der Grunderkrankung zwar quoad vitam offen, hinsichtlich eines Wiedergewinns der verlorenen Teilhabemöglichkeiten aber infaust, wobei im Gespräch eine breite Spanne möglicher verbleibender Teilhabemöglichkeiten sowie die Möglichkeit einer

- Als exemplarische Situationen für eine aktute schwere Erkrankung mit anhaltender Nichteinwilligungsfähigkeit sind insbesondere bei älteren Menschen eine schwer verlaufende Pneumonie und ein schwerer Schlaganfall zu erörtern.

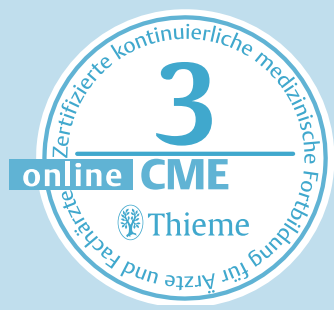


- Um Präferenzen für den Fall eines dauerhaften Verlustes der Einwilligungsfähigkeit zu ermitteln, sollten Fallkonstellationen mit unterschiedlich stark eingeschränkter sozialer Teilhabe durchgespielt werden.
- Unterstützung bei der Auswahl einer geeigneten Vertrauensperson für den Fall eines Verlustes der Einwilligungsfähigkeit.

- Grundsätzlich sind vorausverfügte Behandlungswünsche auch in einer akuten Notfallsituation rechtlich bindend. sukzessiven Verringerung der Teilhabeoptionen im weiteren Krankheitsverlauf zu berücksichtigen sind.

Um Behandlungspräferenzen bei einer Demenz zu ermitteln, sollten unterschiedliche Fallkonstellationen durchgespielt werden, $z$. B.

- eine mittelschwere Demenz mit Restmobilität und mäßig eingeschränktem Sprachvermögen, entsprechend einer noch in gewissem Grad erhaltenen und auch erkennbar genutzten aktiven sozialen Teilhabe sowie

- eine weit fortgeschrittene Demenz mit umfassender Pflegebedürftigkeit mit Bettlägerigkeit und resultierend sehr geringer aktiver sozialer Teilhabe.

\section{Praxistipp}

\section{Lebensfreude}

Auch ist es wichtig, Menschen im Rahmen des Begleitungsgesprächs darauf hinzuweisen, dass viele Demenzkranke noch ausgesprochene Lebensfreude empfinden und zeigen können. Das beizeiten begleiten-Patientenverfügungsformular erlaubt es u. a. festzulegen, dass Bevollmächtigte sich für Behandlungsentscheidungen an dem Kriterium der Lebensfreude orientieren sollen, so weit und so gut sich diese dem Beobachter erschließt.

\section{Auswahl eines Stellvertreters (Bevollmächtigten)}

Ein wesentlicher Bestandteil von BVP ist zudem die Unterstützung bei der Auswahl einer geeigneten Vertrauensperson, die bei Verlust der Einwilligungsfähigkeit stellvertretend für den Betroffenen entscheidet. In manchen Fällen ist es wesentlich, drohende Konflikte (etwa unter mehreren Angehörigen) zu antizipieren und gemeinsam mit allen Beteiligten zu lösen.

Bei der Auswahl eines Bevollmächtigten haben sich folgende Fragen bewährt:

\section{Übersicht}

Fragen zur Auswahl eines Stellvertreters

- Zu wem haben Sie eine tiefe und verlässliche Vertrauensbeziehung?

- Wen erachten Sie für bereit und fähig, gemäß Ihren Wünschen zu entscheiden - ungeachtet eigener Gefühle und Anschauungen?

- Wem trauen Sie am ehesten zu, Ihren Willen z. B. gegenüber Ärzten durchzusetzen, auch gegen Widerstand?

- Wer wohnt in der Nähe, würde sich Zeit nehmen und ist bereit, diese Aufgabe zu übernehmen?

Grundsätzlich ist es möglich, mehrere Personen zu bevollmächtigen.

\section{Praxistipp}

\section{Festlegung mehrerer Vertrauenspersonen}

Dabei ist es aus pragmatischen Gründen empfehlenswert, eine Reihenfolge der Bevollmächtigten festzulegen, verbunden mit dem Hinweis, dass sich die Bevollmächtigten vor wichtigen Behandlungsentscheidungen nach Möglichkeit miteinander besprechen.

\section{Behandlung im Voraus planen: Besonderheiten in Notfallsituationen $\nabla$}

Rechtsverbindlichkeit von Vorausverfügungen in Notfallsituationen

Vorausverfügte Behandlungswünsche sind für ärztliches und nichtärztliches Personal grundsätzlich auch in einer akuten Notfallsituation rechtlich bindend. Dies gilt auch dann, wenn kein gesetzlich vorgesehener Vertreter - Bevollmächtigter oder Betreuer - verfügbar ist, um gemäß $\S 1901$ a BGB die Anwendbarkeit der Vorausverfügung zu prüfen.

Allerdings ist es bei Notfällen oder Krisensituationen für die vor Ort Handelnden oft schwer oder unmöglich zu prüfen, ob eine ausführliche Patientenverfügung auf die vorliegende medizinische Situation zutrifft, da die Prüfung eines detaillierten Textes innerhalb der verfügbaren Sekunden oder Minuten nicht möglich ist und/oder da die darin enthaltenen Festlegungen in der Regel zu allgemein gehalten sowie an prognostische Einschätzungen gebunden sind, welche in der Notfallsituation aber nicht hinreichend verlässlich getroffen werden können. 
Um wirksam werden zu können, müssen Vorausverfügungen demzufolge im Ernstfall vorliegen und auf Anhieb klar ersichtliche Handlungsanweisungen beinhalten. Gut sichtbar aufbewahrte oder - in Institutionen - vom Pflegepersonal bereitgehaltene Notfall- oder Krisenbögen (s.u.) können beide Bedingungen erfüllen.

Eine weitere Schwierigkeit entsteht dadurch, dass in Notfallsituationen oft nichtärztliches Personal - Pflegende in Krankenhäusern und Pflegeeinrichtungen oder Rettungssanitäter - die Erstversorgung übernimmt und damit auch über die Einleitung lebensrettender Sofortmaßnahmen entscheiden muss. Zwar richtet sich das sog. Patientenverfügungsgesetz an den „Arzt“, das verfassungsrechtlich geschützte Recht auf Selbstbestimmung bindet jedoch auch anderes Gesundheitspersonal außerhalb des Geltungsbereichs des §1901a BGB: Eine eindeutig auf die vorliegende Situation zutreffende Vorausverfügung ist folglich auch für nichtärztliches Personal verbindlich, ohne dass dies im Patientenverfügungsgesetz oder im Notfallsanitätergesetz explizit geregelt wäre oder auch nur geregelt sein müsste (ausführlich hierzu [21]).

Anderslautende Vorstellungen (eine Art Pflicht für nichtärztliches Personal zu „reanimieren bis der Arzt kommt") sind in Rettungsdienstkreisen weit verbreitet (s. z. B. [22]) und historisch gut verständlich, da Notfallbögen bis dato in Deutschland keine Verbreitung gefunden haben. Diese Vorstellungen sind jedoch rechtlich nicht haltbar. Rettungsdienstmitarbeiter, die entgegen den in einem unmissverständlich abgefassten Notfallbogen niedergelegten Patientenwillen eine Reanimation beginnen, machen sich prinzipiell der strafbaren Körperverletzung schuldig. Bei der Einführung regionaler BVP-Programme haben entsprechende Fortbildungen für das Personal, aber auch den Träger des Rettungsdienstes daher hohe Priorität.

\section{Merke}

Notfallsanitäter und Pflegende handeln rechtmäßig, wenn sie einer korrekt ausgefüllten ärztlichen Anordnung für den Notfall folgend von einer Reanimation bei Herzstillstand absehen, die der Patient im Voraus für sich ausgeschlossen hat.

\section{Ärztliche Anordnungen für den Notfall}

Die Idee eines Formulars, das eine ärztliche Anordnung über lebensverlängernde Maßnahmen mit der Unterschrift des Patienten (bzw. Vertreters) im Sinne einer Vorausverfügung verbindet, wurde in den 1990er-Jahren in den USA entwickelt; dort sind die Physician Orders for Life-Sustaining Treatment (POLST) vielerorts bundesstaatliche amtliche Formulare (vgl. www.polst.org) und haben in empirischen Untersuchungen ihre Eignung bewiesen [23, 24]. Auch in Deutschland wird seit gut einem Jahrzehnt vermehrt an der Etablierung von Notfall- und Krisenbögen gearbeitet (s. z. B. [25]).

Der Notfallbogen dokumentiert in weitestmöglicher Vereinfachung die gewünschten Behandlungsgrenzen für den Fall einer Krise, die sich aus dem aktuellen Zustand des Betroffenen herausentwickelt. Der Notfallbogen kommt also zum Tragen, wenn der aktuelle, zum Zeitpunkt der Unterschrift gegebene Allgemeinzustand durch eine medizinische Krise akut eingeschränkt ist. Daraus folgt, dass nachhaltige Veränderungen des Allgemeinzustands oder der Behandlungswünsche eine Überprüfung und ggf. Anpassung des Notfall- und Krisenbogens nach sich ziehen sollten.

Der Notfallbogen gibt zunächst dem Arzt die Möglichkeit, in Absprache mit dem Patienten oder seinem Vertreter diejenigen Behandlungsoptionen auszuschließen, die medizinisch nicht indiziert sind (weil für das gegebene Behandlungsziel nicht mehr Erfolg versprechend). Darüber hinaus definiert der Notfallbogen die vom Patienten (bzw. vom Vertreter im Namen des Patienten) festgelegten, individuell gewünschten Behandlungsgrenzen, die im Notfall gelten sollen.

Die Erfahrung zeigt, dass die allermeisten gesunden Menschen im berufstätigen Alter im Notfall keine Einschränkung einer medizinisch indizierten, lebensrettenden Notfalltherapie wünschen. Im höheren Alter und/oder bei fortschreitenden chronischen Einschränkungen verliert der Wunsch, noch lange zu leben, hingegen oft (aber keineswegs immer!) an Gewicht gegenüber dem Wunsch, einen „guten Tod“ sterben und (weiteren) chronischen Einschränkungen der sozialen Teilhabemöglichkeit entgehen zu können.

Der Notfallbogen wird durch die Unterschrift des Betroffenen (bzw. Angehörigen) zu einer Patientenverfügung (bzw. Festlegung des Vertreters) im Sinne des Patientenverfügungsgesetzes. Die zusätzliche Unterschrift des behandelnden Arztes attestiert für das in der Krisensituation handelnde Gesundheitspersonal Einwilligungsfähigkeit und Verständnis der Implikationen seitens des Patienten, somit die Validität der Verfügung.
- Notfall- oder Krisenbögen müssen schnell verfügbar sein und auf Anhieb klar ersichtliche Handlungsanweisungen liefern.

- Rettungsdienstmitarbeiter begehen eine (potenziell strafbare!) Körperverletzung, wenn sie entgegen dem im ihnen vorliegenden Notfallbogen niedergelegten Patientenwillen mit einer Reanimation beginnen.

- Der Notfallbogen dokumentiert in weitestmöglicher Vereinfachung die gewünschten Behandlungsziele und -grenzen für den Fall einer akuten medizinischen Krise mit Verlust der Einwilligungsfähigkeit.

- Die Unterschrift des behandelnden Arztes bestätigt die Validität der Notfallplanung. 
(Haus-)Ärztliche Anordnung für den Notfall (HAnNo) im Projekt beizeiten begleiten Ein für die regionale Verwendung konzipierter Notfallbogen ist die (Haus-)Ärztliche Anordnung für den Notfall (HAnNo), die für das Modellprojekt beizeiten begleiten entwickelt wurde [26, 27]. Die HAnNo ist Bestandteil der Patientenverfügung und regelt explizit nur das notfallmäßige Vorgehen im Fall einer lebensbedrohlichen Krise. Aus diesem Grund werden in der HAnNo - anders als in den in den USA verbreiteten POLST-Formularen - u.a. weder die Frage einer PEG-Sonde noch die der Gabe von Antibiotika behandelt.

Der Optionsteil zeichnet sich in formaler Hinsicht dadurch aus, dass (anders als in den gängigen US-POLST-Formularen) nur genau ein Kreuz gesetzt werden kann; dadurch werden auf POLSTund manchen deutschen Notfallbögen mögliche, aber klinisch unsinnige Kombinationen (z.B. Reanimation ja, aber Krankenhauseinweisung nein) ausgeschlossen.

Inhaltlich gibt es 3 Kategorien (A, B und C), die am Therapieziel und bei den Optionen der Kategorie $B$ zudem an den Belastungen und Risiken klinisch relevanter Behandlungsoptionen orientiert sind (die HAnNo finden Sie online unter: http://dx.doi.org/10.1055/s-0042-110711).

\section{Übersicht}

Die 3 Kategorien der (Haus-)Ärztlichen Anordnung für den Notfall (HAnNo) im Projekt beizeiten begleiten

- Option A entspricht dem uneingeschränkten Einsatz aller medizinisch vertretbaren und indizierten Mittel zur Erreichung des Therapieziels der Lebensverlängerung und damit dem gängigen akutmedizinischen Standard, der grundsätzlich auch dann zur Anwendung kommt, wenn keine Vorausverfügung vorliegt.

- Option B bejaht ebenfalls die Orientierung am Therapieziel der Lebensverlängerung, jedoch „nicht um jeden Preis“: Vielmehr besteht die Möglichkeit, 4 klinisch besonders relevante Behandlungen kumulativ auszuschließen, die durch spezielle, im Voraus gut zu würdigende Risiken bzw. qualitative Prognosesprünge gekennzeichnet sind:

$\checkmark$ die kardiopulmonale Reanimation (BO),

zusätzlich die invasive Beatmung über einen Tubus (mit einer im Unterschied zur von diesem Ausschluss nicht berührten nicht-invasiven Beatmung deutlich schlechteren Prognose - B1),

zusätzlich die Behandlung auf Intensivstation (B2) sowie

zusätzlich jegliche stationäre Einweisung mit dem Ziel der Lebensverlängerung (B3).

- Option C unterscheidet sich grundsätzlich von den beiden vorgenannten Optionen, indem das Therapieziel der Lebensverlängerung durch dasjenige der Linderung von Beschwerden (Palliation) explizit ersetzt wird. Die Auswahl dieser Option kann dadurch begründet sein, dass der Versuch einer Lebensverlängerung medizinisch nicht indiziert wäre, oder dadurch, dass eine medizinisch mögliche (,indizierte“) Lebensverlängerung vom Patienten nicht mehr gewünscht wird.

Bei konkreten, begründeten Zweifeln an der Anwendbarkeit des Notfallbogens gilt: In dubio pro vita.

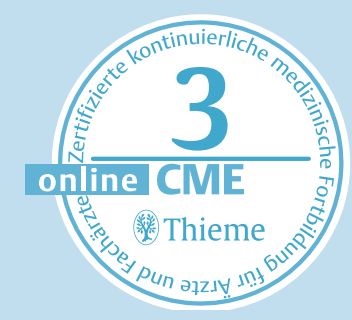

Abschließend ist darauf hinzuweisen, dass auch die HAnNo nicht jeden (konstruierten) Anwendungsfall vollständig abdeckt - das ist auch nicht der Anspruch. In den seltenen Zweifelsfällen (etwa auch bei konkreten, aber nicht klar dokumentierten Hinweisen auf einen zwischenzeitlich geänderten Patientenwillen) gilt auch hier: In dubio pro vita.

\section{Merke}

Ein Notfallbogen kann nur so gut bzw. erfolgreich sein wie die regionale Implementierung des BVP-Programms, dessen Teil er ist, und das bedeutet: wie die Schulung und Mitwirkung aller beteiligten Akteure, insbesondere der BVP-Gesprächsbegleiter und in der Folge der von ihnen moderierte Gesprächsprozess.

\section{Möglichkeiten der Vorausplanung für nicht einwilligungsfähige Personen \\ $\nabla$}

Behandlung im Voraus planen (BVP) ist primär dafür konzipiert, mit einwilligungsfähigen Menschen mögliche zukünftige medizinische Entscheidungen bei Verlust der Einwilligungsfähigkeit vorauszuplanen. Aktuell gibt es aber gerade in stationären Pflegeeinrichtungen und Einrichtungen der Eingliederungshilfe für behinderte Menschen eine große Anzahl von Menschen, die

- keine Patientenverfügung verfasst,

- ihre Einwilligungsfähigkeit verloren haben, oder

- nie einwilligungsfähig waren. 
Behandlungsentscheidungen für diese Menschen werden grundsätzlich von ihren Vertretern getroffen.

Insbesondere in Notfallsituationen sind diese meist nicht verfügbar bzw. von der Situation überfordert, sodass ohne eine Vorausplanung erfahrungsgemäß die akutmedizinischen Standards der uneingeschränkten lebensverlängernden Therapie greifen - unabhängig davon, ob dies noch dem (mutmaßlichen) Patientenwillen entspricht. Bislang gibt es in Deutschland für diese Situationen kein offiziell etabliertes Instrument der Vorausplanung. Dennoch werden in der Praxis de facto Entscheidungen für nicht einwilligungsfähige Personen vorausgeplant. In einer eigenen Vollerhebung von Vorausverfügungen in einer deutschen Großstand waren immerhin $11 \%$ der Verfügungen nicht vom Betroffenen selbst, sondern von einem Vertreter unterschrieben [7].

Um diese in Pflegeeinrichtungen offenbar weitverbreitete, aber bisher intransparente Form der Vorausplanung sichtbar und dadurch Qualitätsstandards zugänglich zu machen, wurde im BVPPilotprojekt beizeiten begleiten das Instrument der Vertreterverfügung entwickelt (ausführlicher in [28]). Unter einer Vertreterverfügung wird ein schriftliches Dokument verstanden, das nicht von der betroffenen, nicht einwilligungsfähigen Person, sondern von ihrem Vertreter unterzeichnet worden ist und in dem medizinische Behandlungsentscheidungen für den hypothetischen Fall künftiger gesundheitlicher Krisensituationen im Voraus festgelegt werden.

Ein dazu legitimierter Vertreter ist der Legaldefinition nach

- entweder ein von der betreffenden Person in früherer Zeit autorisierter Bevollmächtigter

(vgl. §1896 Abs. 2 Satz 2, §1901a Abs. 5 BGB)

- oder aber ein vom Gericht nach $\S 1896$ Abs.1 BGB bestellter Betreuer für den Bereich der Gesundheitsfürsorge.

Die Vertreterverfügung muss als notwendige Voraussetzung einer patientenzentrierten Medizin bei nicht einwilligungsfähigen Personen angesehen werden, weil nur durch sie gewährleistet werden kann, dass dauerhaft nicht einwilligungsfähige Menschen, für die keine diesbezüglich aussagekräftige Patientenverfügung vorliegt, in künftigen hypothetischen Notfällen 1. medizinisch sinnvoll und 2. ihren individuellen Bedürfnissen und Behandlungswünschen entsprechend behandelt werden.

Aber auch für nicht akute Entscheidungssituationen erscheint eine Vorausplanung mit dem Vertreter sinnvoll, da dieser frühzeitig und ohne den akuten Entscheidungsdruck überlegen kann, welche Behandlungen den mündlich geäußerten Wünschen oder dem mutmaßlichen Willen des Patienten entsprechen. Auf diese Vorausplanungen kann auch dann zurückgegriffen werden, wenn der Vertreter selbst erkrankt oder sogar vor dem Betroffenen verstorben ist.

Nicht zuletzt kann die Vertreterverfügung dazu dienen, eine früher vom Betroffenen in einer eigenhändigen Patientenverfügung getroffene Festlegung zu gegebener Zeit umzusetzen: Etwa wenn aktuell infolge wiederholter Schlaganfälle ein Zustand schwerster Demenz eintritt, für den der Betroffene früher den Verzicht auf jegliche lebensverlängernden Maßnahmen verfügt hat und der Vertreter jetzt eine entsprechende Notfallverfügung unterzeichnet, die im Sinne des Betroffenen u. a. den Verzicht auf einen Wiederbelebungsversuch bei Herzstillstand beinhaltet.

Die beizeiten begleiten-Vertreterverfügung bietet dem Vertreter zu Beginn die Möglichkeit, die Quelle der Festlegungen - z. B. früher mündlich geäußerte Behandlungswünsche, ein mutmaßlicher Patientenwille oder Überlegungen zum Wohlergehen des Patienten - zu benennen und mit möglichst konkreten Aussagen bzw. Hinweisen zu belegen.

Analog zur Patientenverfügung sollte eine Vertreterverfügung dann Informationen und Festlegungen zu den in der Infobox zusammengefassten Themen und Szenarien enthalten.

\section{Übersicht}

\section{Informationen und Festlegungen einer Vertreterverfügung}

- Allgemeine Einstellungen des Betroffenen zum (Weiter-)Leben und Sterben unter den aktuellen Lebensbedingungen sowie ggf. Hinweise auf Bedingungen oder Szenarien, unter denen der Betroffene mutmaßlich eine Änderung des Therapieziels zur reinen Leidenslinderung (Palliation) gewünscht hätte.

- Die mündlich geäußerten oder mutmaßlichen Wünsche des Betroffenen zu lebensrettenden bzw. -verlängernden Behandlungsmaßnahmen, und zwar sowohl zu notfallmedizinischen Interventionen (zu dokumentieren auf einem separaten Notfallbogen) als auch zu nichtakuten Entscheidungen über lebensverlängernde Maßnahmen im weiteren Verlauf der Erkrankung.

In einer Vertreterverfügung sollte immer angegeben werden, auf welcher Quelle die Festlegungen beruhen: früher mündliche geäußerte Behandlungswünsche, ein mutmaßlicher Patientenwille oder Überlegungen zum Wohlergehen des Patienten.

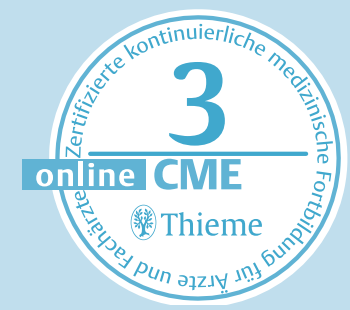


- Die Vertreterverfügung kann wie die Patientenverfügung jederzeit widerrufen oder geändert werden. Sie ist bindend für andere Personen (Behandler), nicht jedoch für den Vertreter.
Das Patientenverfügungsgesetz sieht eine schriftliche Vorausplanung durch den Vertreter („Vertreterverfügung“) nicht ausdrücklich vor. Wie bereits oben ausgeführt, ist das Gesetz jedoch nicht als eine abschließende Regelung zu verstehen.

Die rechtliche Bewertung hängt folglich wesentlich davon ab, ob Vertreterverfügungen geeignet sind, den Vertreter in der Erfüllung des ihm zugewiesenen Auftrags, nämlich der Entscheidung über medizinische Eingriffe im Sinne eines dauerhaft nicht einwilligungsfähigen Betroffenen, zu unterstützen. Alles spricht dafür, dass dies mit einer rechtzeitigen Vorbereitung auf künftige Geschehnisse insbesondere durch die verlässliche Gewinnung, Sammlung und Sicherung von Informationen z.B. über frühere mündliche oder schriftliche Äußerungen, ethische oder religiöse Überzeugungen und sonstige persönliche Wertvorstellungen (vgl. §1901a Abs.2 Satz3 BGB) bestmöglich zu gewährleisten ist.

\section{Merke}

Der Vertreter bindet mit seiner Verfügung stets nur andere, nie sich selbst, ähnlich wie eine Patientenverfügung vom Betroffenen jederzeit widerrufen werden kann (vgl. §1901a Abs. 1 Satz3 BGB).

Auf diese Besonderheit muss in einer BVP-Gesprächsbegleitung unbedingt hingewiesen werden, um Missverständnisse zu vermeiden; sie sollte auch auf den entsprechenden Formularen vermerkt und nicht zuletzt den Behandelnden im Rahmen der regionalen Implementierung eines BVP-Programms bekanntgemacht worden sein.

\section{Beispiel}

Denkbar wäre z. B., dass der Vertreter für den Fall einer medizinischen Krise einer stationären Einweisung mit dem Ziel der Lebensverlängerung im Sinne des Betroffenen prinzipiell zustimmt, da er die Chancen einer Notfalleinweisung pauschal höher bewertet als deren Belastungen und Risiken. Andererseits ist es jedoch auch möglich, dass er in einem konkreten Einzelfall, bei dem er rechtzeitig vor Ort sein kann, gemeinsam mit dem behandelnden Arzt zu dem Schluss kommt, dass der Betroffene angesichts der hier gegebenen Konstellation von Chancen und Risiken eine Einweisung nicht wollen würde.

\section{Implementierung in der Region durch Vernetzung und Change-Management} $\nabla$

Behandlung im Voraus planen ist ein systematisches Konzept für die regionale Umstrukturierung eines Gesundheitswesens mit dem Ziel, einer patientenzentrierten Versorgung stärkeres Gewicht zu verleihen, insbesondere für Behandlungen bei aktueller oder dauerhafter Nichteinwilligungsfähigkeit. Dieser Systemwandel ist von einzelnen Akteuren kaum zu leisten. Es bedarf vielmehr der konzertierten Anstrengung eines regionalen Teams, dem Leitungen bzw. Führungskräfte verschiedener Bereiche angehören sollten, insbesondere der in der Übersicht zusammengefasste Personenkreis.

\section{Übersicht}

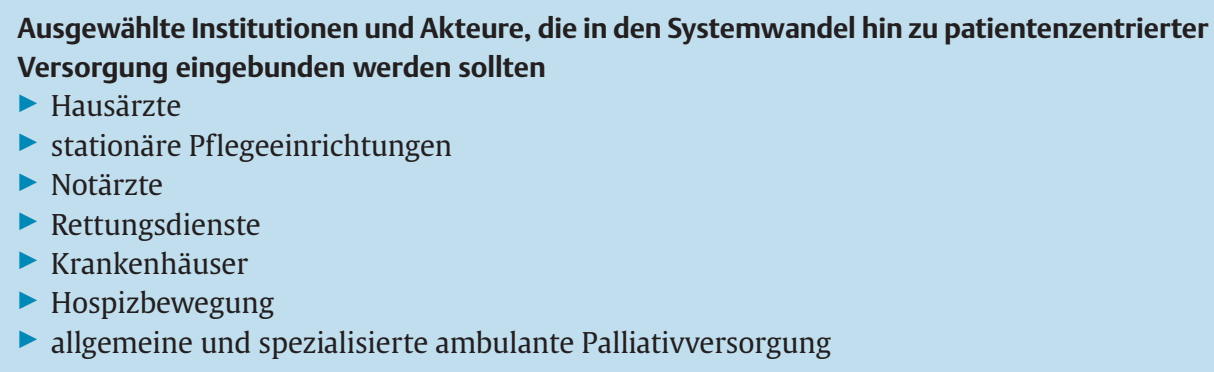

Neben der Netzwerkbildung sind die spezifischen BVP-Rollen zu beachten. Im Zentrum steht der nichtärztliche BVP-Gesprächsbegleiter, der, wo immer möglich, in Kooperation mit hierfür zertifizierten Hausärzten tätig wird. Daneben werden naturgemäß BVP-Gesprächsbegleiter-Trai- 
ner benötigt. Für eine Sensibilisierung der Öffentlichkeit sind BVP-Botschafter wünschenswert, die durch Vorträge etc. Basisinformationen vermitteln und dazu anregen, über das Thema (und konkret die eigenen Behandlungspräferenzen) nachzudenken. Zudem braucht eine Region einen verantwortlichen Projektkoordinator, der die Qualifizierungsprozesse, die Netzwerkbildung sowie Maßnahmen der Qualitätssicherung vorantreibt (nach unserer, auf internationale Vorbilder gestützten Einschätzung ca. 1/4 bis 1/2 Stelle auf 200000 Einwohner).

Zu Beginn der regionalen Implementierung eines BVP-Programms wie beizeiten begleiten sollte nur mit Institutionen und Personen zusammengearbeitet werden, die von der Notwendigkeit und Sinnhaftigkeit des Systemwandels überzeugt sind und das gerade in der Anfangsphase erforderliche Engagement mitbringen. Unabdingbar ist zudem die vorbehaltlose Unterstützung des Vorhabens durch die jeweiligen Leitungspersonen.

\section{Praxistipp}

Auch ein kleines multiprofessionelles Team, das z. B. aus 3 Pflegeeinrichtungen, den darin am stärksten präsenten 5 Hausärzten, dem zugehörigen Rettungsdienst und dem internistischen Chefarzt des wichtigsten Einzugskrankenhauses bestehen könnte, kann erfolgreich eine „regionale“ Implementierung starten.

Die Implementierung eines BVP-Programms wird von den Mitarbeitern wie auch den Patienten meist dankbar angenommen und entfaltet häufig eine Sogwirkung auf die umliegenden Institutionen und Strukturen.

Bei der Einführung regional einheitlicher Formulare und der entsprechenden Qualifizierung der Gesprächsbegleiter ist darauf zu achten, dass Standards definiert und eingehalten werden. Jeder einzelne Gesprächsbegleiter muss vom Projektkoordinator bzw. Trainer im Rahmen supervidierter Gesprächsbegleitungen zertifiziert werden.

\section{Merke}

Es dürfen nur solche Fachkräfte zertifiziert werden, die zu einer patientenzentrierten Gesprächsbegleitung ohne suggestive oder gar manipulative Elemente bzw. ohne „eigene Agenda“ fähig sind.

\section{Ausblick: Behandlung im Voraus planen in deutschen Pflegeeinrichtungen gemäß §132 g SGB V \\ $\nabla$}

Mit dem Ende 2015 in Kraft getretenen Hospiz- und Palliativgesetz (HPG) werden nun die Voraussetzungen für eine bundesweite Etablierung von BVP geschaffen [29]. Der neue §132g SGB V „Gesundheitliche Versorgungsplanung für die letzte Lebensphase“ ermöglicht den vollstationären Pflegeeinrichtungen und Einrichtungen der Eingliederungshilfe für behinderte Menschen, ihren Bewohnern eine gesundheitliche Versorgungsplanung durch nicht-ärztliche Gesprächsbegleiter anzubieten - zulasten der Krankenkasse des Versicherten. Ausdrücklich sind dabei der behandelnde Hausarzt, für den ebenfalls eine spezifische Vergütung vorgesehen ist, und andere regionale Betreuungs- und Versorgungsangebote einzubeziehen, mit Blick auf mögliche Notfallsituationen insbesondere auch Rettungsdienste und Krankenhäuser.

Bis Ende 2016 will der GKV-Spitzenverband dem gesetzlichen Auftrag entsprechend mit den Vereinigungen der Träger der Pflegeeinrichtungen die weiteren Details zu Inhalt und Anforderungen der gesundheitlichen Versorgungsplanung vereinbaren.

Zur Unterstützung dieser Verhandlungen erarbeitet eine an der Deutschen Gesellschaft für Palliativmedizin angesiedelte, vom Bundesministerium für Gesundheit geförderte Task Force mit Vertretern einschlägiger Fachgesellschaften und Berufsgruppen seit diesem Frühjahr Qualitätsstandards für die Implementierung von BVP und insbesondere die BVP-Gesprächsbegleitung. Dabei werden auch BVP-Muster-Curricula für die Qualifizierung der Gesprächsbegleiter und deren Trainer sowie für kooperierende (Haus-)Ärzte, Empfehlungen für die Schulung und Einbindung der relevanten Institutionen und Akteure des regionalen Versorgungssystems sowie geeignete Evaluationskonzepte für dieses neuartige Element im Gesundheitswesen entwickelt. Bei der Umsetzung des $\S 132 \mathrm{~g}$ SGB V sind verschiedene Herausforderungen zu bewältigen:

- BVP als neues Konzept ist bislang in Fachkreisen und in der Öffentlichkeit wenig bekannt, und der für die regionale Implementierung von BVP erforderliche kulturelle Wandel hin zu einer

- Voraussichtlich ab Anfang 2017 können bundesweit Pflegeeinrichtungen und Einrichtungen der Eingliederungshilfe für behinderte Menschen ihren Bewohnern eine krankenkassenfinanzierte "gesundheitliche Versorgungsplanung", d. h. BVP anbieten.

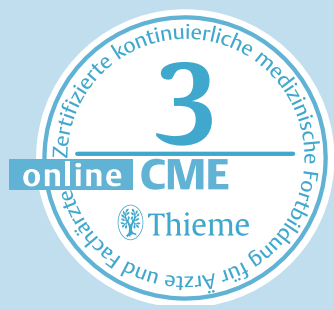


Bislang ist die Vergütung von BVP durch das Hospiz- und Palliativgesetz (HPG) auf stationäre Pflegeeinrichtungen und Einrichtungen der Eingliederungshilfe für behinderte Menschen beschränkt. Engagierte Netzwerke und Institutionen bzw. Akteure können ungeachtet dessen eine BVP-Initiative starten bzw. sich an geförderten Projekten beteiligen.

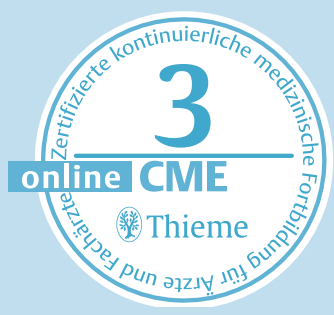

patientenorientierten Ermittlung und regelhaften Beachtung von Patientenwünschen dürfte den internationalen Erfahrungen zufolge nicht von heute auf morgen zu bewältigen sein.

- Bislang fehlen entsprechend qualifizierte Trainer für die Ausbildung der erforderlichen großen Anzahl von BVP-Gesprächsbegleitern.

- Zudem sieht das Gesetz bislang keine Ressourcen für eine regionale Projektkoordination vor, die aber gerade in der Anfangszeit als eine notwendige Voraussetzung für die effektive Implementierung von BVP-Programmen im regionalen Versorgungssystem erscheint.

Angesichts der überzeugenden Argumente für BVP mag man bedauern, dass das HPG die Einführung von BVP auf stationäre Pflegeeinrichtungen und Einrichtungen der Eingliederungshilfe für behinderte Menschen beschränkt. Angesichts des heutigen Entwicklungsstands von BVP in Deutschland erscheint dies aber ein realistischer und politisch kluger erster Schritt zu sein; weitere Schritte dürften folgen, wenn die Evaluierung der hier gemachten Erfahrungen dies nahelegt. Es bleibt darüber hinaus einzelnen engagierten Netzwerken, Institutionen und Akteuren unbenommen, auch ohne separate Förderung gemäß §132 g SGB V eine BVP-Initiative zu starten und dabei nach Möglichkeit - wie im HPG ausdrücklich vorgesehen - eine Vernetzung mit den regionalen Versorgern anzustreben. Insbesondere wäre es wünschenswert, wenn die in $\S 132 \mathrm{~g}$ SGB V, Abs. 2 ausdrücklich hervorgehobenen Hausärzte hierbei eine Führungsrolle übernehmen würden, da bei ihnen die fachliche Verantwortung für den kontinuierlichen, prinzipiell lebenslangen Prozess der Vorausplanung am besten aufgehoben zu sein scheint.

Kernaussagen

- Behandlung im Voraus planen (BVP) bzw. Advance Care Planning (ACP) verfolgt die übergreifende Zielsetzung, dass Patienten auch dann nach ihren individuellen Wünschen behandelt werden, wenn sie diese selbst nicht mehr äußern können.

- Mit der konventionellen Herangehensweise an Patientenverfügungen ist dieses Ziel nicht zu erreichen: Diese sind nach wie vor zu wenig verbreitet, häufig nicht aussagekräftig formuliert und von fragwürdiger Validität, bei Bedarf oft nicht zur Hand, und sie bleiben vom Gesundheitspersonal oft unbeachtet.

- Um die übergreifende Zielsetzung zu erreichen - effektive Wahrung der Patientenautonomie bei Verlust der Einwilligungsfähigkeit -, weisen BVP-Programme 2 konstitutive Elemente auf:

- eine qualifizierte Gesprächsbegleitung im Sinne einer gemeinsamen Entscheidungsfindung und

$\checkmark$ eine regionale Implementierung.

- Im Rahmen eines professionell begleiteten Gesprächsprozesses erhalten Menschen Gelegenheit, eigene Präferenzen für mögliche zukünftige medizinische Behandlungen bei Nichteinwilligungsfähigkeit zu entwickeln und auf aussagekräftigen Formularen zu dokumentieren.

- Die in den regionalen Versorgungsstrukturen tätigen Personen werden so geschult bzw. informiert, dass die resultierenden Vorausverfügungen regelmäßig verfügbar sind und zuverlässig beachtet werden.

- Internationale Studien belegen, dass mittels umfassender BVP-Programme nicht nur die Selbstbestimmung von Patienten in der letzten Lebensphase effektiv berücksichtigt, sondern auch die Qualität der Versorgung für Patienten und ihre Angehörigen verbessert werden kann.

- Der neue §132g SGB V verschafft nun zunächst einmal den stationären Pflegeeinrichtungen Deutschlands sowie kooperierenden Institutionen und Akteuren die Möglichkeit, ihren Bewohnern eine BVP zulasten der Krankenkassen anzubieten.

\section{Hinweis}

Der vorliegende Beitrag enthält wesentliche Teile aus: in der Schmitten J, Marckmann G. Vorausschauende Behandlungsplanung. In: Marckmann G, Hrsg. Praxisbuch Ethik in der Medizin. Berlin: Medizinisch Wissenschaftliche Verlagsgesellschaft; 2015: 53-66. Mit freundlicher Genehmigung der Medizinisch Wissenschaftlichen Verlagsgesellschaft.

\section{Interessenkonflikt}

$\nabla$

Die Autoren sind als BVP-Gesprächsbegleiter-Trainer tätig und unterstützen Institutionen oder Regionen, die BVP einführen wollen, beim erforderlichen Change Management. Darüber hinaus erklären die Autoren, dass keine Interessenkonflikte vorliegen. 
1 Coors M, Jox RJ, in der Schmitten J, Hrsg. Advance Care Planning. Von der Patientenverfügung zur gesundheitlichen Vorausplanung. Stuttgart: Kohlhammer; 2015

2 Beauchamp TL, Childress JF. Principles of biomedical ethics. 7th: edn. Oxford: Oxford University Press; 2013

3 Katzenmeier C. Ärztliche Aufklärung. In: Wiesemann C, Simon A, Hrsg. Patientenautonomie Theoretische Grundlagen - Praktische Anwendungen. Münster: Mentis; 2013: 91 -105

4 Ach J, Schöne-Seiffert B. „Relationale Autonomie“ - eine kritische Analyse. In: Wiesemann C, Simon A, Hrsg. Patientenautonomie Theoretische Grundlagen - Praktische Anwendungen. Münster: Mentis; 2013: 42 60

5 Brock $D$. The ideal of shared decision making between physicians and patients. Kennedy Inst Ethics J 1991; 1: $28-48$

6 in der Schmitten J. Autonomie gewähren genügt nicht - Patienten-Selbstbestimmung bedarf aktiver Förderung durch Ärzte. Z Allgemeinmed 2014; 90: 246 - 250

7 Sommer S, Marckmann G, Pentzek $M$ et al. Patientenverfügungen in stationären Einrichtungen der Seniorenpflege. Vorkommen, Validität, Aussagekraft und Beachtung durch das Pflegepersonal. Dtsch Ärztebl 2012; 109: $577-583$

8 Schoffner M, Schmidt KW, Benzenhofer $U$ et al. Patientenverfügung auf dem Prüfstand: Ärztliche Beratung ist unerlässlich. Dtsch Med Wochenschr 2012; 137: 487-490

9 Nauck F, Becker M, King $C$ et al. To what extent are the wishes of a signatory reflected in their advance directive: a qualitative analysis. BMC Med Ethics 2014; 15: 52

10 Fagerlin A, Schneider CE. Enough. The failure of the living will. Hastings Cent Rep 2004; 34: 30 - 42

11 Hammes BJ, Briggs LA. Das „Respecting Choices“ Advance-Care-Planning-Programm in den USA: ein nachgewiesener Erfolg. In: Coors M, Jox RJ, in der Schmitten J, Hrsg. Advance Care Planning: Von der Patientenverfügung zur gesundheitlichen Vorausplanung. Stuttgart: Kohlhammer; 2015: 181-195

12 Hammes BJ, Rooney BL, Gundrum JD. A comparative, retrospective, observational study of the prevalence, availability, and specificity of advance care plans in a county that implemented an advance care planning microsystem. J Am Geriatr Soc 2010; 58: 1249-1255

13 Detering K, Silvester W. Implementierung von Advance Care Planning in Australien: das Programm „Respecting Patient Choices“. In: Coors M, Jox RJ, in der Schmitten J, Hrsg. Advance Care Planning: Von der Patientenverfügung zur gesundheitlichen Vorausplanung. Stuttgart: Kohlhammer; 2015: 196-206

14 Detering KM, Hancock $A D$, Reade $M C$ et al. The impact of advance care planning on end of life care in elderly patients: randomised controlled trial. BMJ 2010; 340: c1345

15 Manson L, Muir S, Snow B. Das Erfolgsmodell „Our voice - to tatou reo“: Advance Care Planning in Neuseeland. In: Coors M, Jox RJ, in der Schmitten J, Hrsg. Advance Care Planning: Von der Patientenverfügung zur gesundheitlichen Vorausplanung. Stuttgart: Kohlhammer; 2015: 207-224

16 Schulze U, Niewohner S. Selbstbestimmt in der letzten Lebensphase - zwischen Autonomie und Fürsorge. Impulse aus dem Modellprojekt LIMITS Münster. Münster: Lit Verlag Münster; 2004

17 in der Schmitten J, Marckmann G. Das Pilotmodell beizeiten begleiten. In: Coors M, Jox R, in der Schmitten J, Hrsg. Advance Care Planning: Von der Patientenverfügung zur gesundheitlichen Vorausplanung. Stuttgart: Kohlhammer; 2015: 234-257

18 in der Schmitten J, Lex K, Mellert C et al. Patientenverfügungsprogramm - Implementierung in Senioreneinrichtungen: Eine interregional kontrollierte Interventionsstudie. Dtsch Ärztebl 2014; 111: 50 - 57

19 Krones T, Otto T, Karzig I et al. Advance Care Planning im Krankenhaussektor - Erfahrungen aus dem Züricher 'MAPS' Trial. In Advance Care Planning - von der Patientenverfügung zur gesundheitlichen Vorausplanung. Stuttgart: Kohlhammer; 2015: 270-287

20 Beyer S, Ertl R, Bitschnau K. Dialog als Vorsorge - Ist die Vorbereitung auf die Krisensituation in Pflegeheimen möglich? Erfahrungen aus Österreich. In: Coors M, Jox R, in der Schmitten J, Hrsg. Advance Care Planning: Von der Patientenverfügung zur gesundheitlichen Vorausplanung. Stuttgart: Kohlhammer; 2015: $225-233$

21 in der Schmitten J, Rixen S, Marckmann G. Vorausplanung in der Nofall- und Intensivmedizin. In: Coors M, Jox RJ, in der Schmitten J, Hrsg. Advance Care Planning: Von der Patientenverfügung zur gesundheitlichen Vorausplanung. Stuttgart: Kohlhammer; 2015: 288-301

22 Wiese CHR, Ittner BM, Graf BM et al. Palliative Notfälle - Definition, Besonderheiten und therapeutische Entscheidungen. Notarzt 2011; 27: 223-226

23 Hammes BJ, Rooney BL, Gundrum JD et al. The POLST program: a retrospective review of the demographics of use and outcomes in one community where advance directives are prevalent. J Palliat Med 2012; 15: $77-85$

24 Hickman SE, Nelson CA, Moss AH et al. The consistency between treatments provided to nursing facility residents and orders on the physician orders for life-sustaining treatment form. J Am Geriatr Soc 2011; 59: 2091 - 2099

25 Wiese $\mathrm{CH}$, Bartels $U$, Geyer A et al. Gottinger Palliativkrisenbogen: Verbesserung der notfallmedizinischen Versorgung von ambulanten Palliativpatienten. Die „Gelbe Karte fur den Rettungsdienst“. Dtsch Med Wochenschr 2008; 133: 972 - 976

26 in der Schmitten J, Rothärmel S, Rixen S et al. Patientenverfügungen im Rettungsdienst (Teil 2). Neue Perspektiven durch Advance Care Planning und die „Hausärztliche Anordnung für den Notfall“. Notfall Rettungsmed 2011; 14: $465-474$

27 in der Schmitten J, Rixen S, Marckmann G. Vorausplanung in der Notfall- und Intensivmedizin. In: Coors M Jox R, in der Schmitten J, Hrsg. Advance Care Planning: von der Patientenverfügung zur gesundheitlichen Vorausplanung. Stuttgart: Kohlhammer; 2015: 288-301

28 in der Schmitten J, Jox RJ, Rixen S et al. Vorausplanung für nicht-einwilligungsfähige Personen - „Vertreterverfügungen“. In: Coors M, Jox RJ, in der Schmitten J, Hrsg. Advance Care Planning: Von der Patientenverfügung zur gesundheitlichen Vorausplanung. Stuttgart: Kohlhammer; 2015: 119-140

29 Rixen S, Marckmann G, in der Schmitten J. Gesundheitliche Versorgungsplanung für die letzte Lebensphase - Das Hospiz- und Palliativgesetz. Neue Jur Wochenschr 2016; 69: 125-129

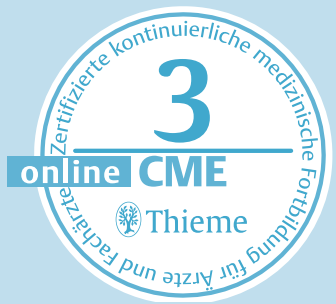




\section{CME-Fragen Behandlung im Voraus planen (Advance Care Planning): ein neues Konzept zur Realisierung wirksamer Patientenverfügungen}

1 Welche Aussage trifft nicht zu? Patientenverfügungen erfüllen nur dann ihren Zweck, effektive Wahrung der Selbstbestimmung bei Nichteinwilligungsfähigkeit zu ermöglichen, wenn sie...

A ... aussagekräftig für relevante klinische Szenarien formuliert wurden (Aussagekraft).

B ... in ausreichender Vielfalt erstellt wurden (Diversität).

C ...verlässlich den wohlinformierten Willen der Betroffenen wiedergeben (Validität).

D ...in der Entscheidungssituation tatsächlich vorliegen (Verfügbarkeit).

E .... angemessen vom Gesundheitspersonal bei der Behandlungsentscheidung beachtet werden (Beachtung).

Das Konzept Behandlung im Voraus planen (BVP) verfolgt eines der im Folgenden genannten Ziele nicht. Welches? mögliche künftige medizinische Entscheidungen vorauszuplanen, um Patienten zuverlässig nach ihren individuellen Wertvorstellungen und Wünschen behandeln zu können

B mögliche künftige medizinische Entscheidungen mit Stellvertretern für Patienten vorauszuplanen, die ihren Willen krankheitsbedingt bereits nicht mehr selbst äußern können oder auch niemals äußern konnten

C auch Wünsche und Präferenzen zu anderen künftig relevanten Aspekten der Versorgung und Begleitung äußern und festlegen zu können

D nicht einwilligungsfähige Patienten vor ungewollter Überoder Unterversorgung zu bewahren

E Bevollmächtigte/Betreuer sowie Ärzte von jeglicher stellvertretenden Entscheidung zu entbinden

Die Zielsetzung von Behandlung im Voraus planen (BVP) wird durch welche der im Folgenden genannten Interventionen nicht erreicht?

A durch vorweggenommene gemeinsame Entscheidungsfindung (Shared Decision Making)

B durch Ausgabe optimierter Formulare an Menschen, die eine Patientenverfügung erstellen wollen

C auf individueller Ebene durch einen qualifizierten, professionell begleiteten Gesprächsprozess

D durch die Gelegenheit, eigene Präferenzen für künftige medizinische Behandlung bei Verlust der Einwilligungsfähigkeit zu entwickeln

E auf der Systemebene durch die Einbindung aller relevanten regionalen Institutionen und Versorgungsstrukturen
Welche Aussage trifft zu? Behandlung im Voraus planen (BVP) beinhaltet regelmäßig...

A ... eine schriftliche Beurkundung der vorausverfügten Wünsche durch einen Anwalt/Juristen.

B ... Entscheidungen zum Abbruch lebenserhaltender Maßnahmen.

C ... ausschließlich Hinweise zu Maßnahmen, die bei einer Nichteinwilligungsfähigkeit der Betroffenen unterlassen werden sollen.

D ..., dass die resultierenden Vorausverfügungen verfügbar sind und zuverlässig respektiert werden.

E ... die Zusammenarbeit mit professionellen Ethikern.

5 Eine der folgenden Maßnahmen gehört nicht zu den wichtigsten Elementen eines BVP-Programms. Welche?

A ein aufsuchendes Gesprächsangebot und Freiwilligkeit der Teilnahme

B eine qualifizierte Gesprächsbegleitung und kontinuierliche Qualitätssicherung

C eine vertragliche Verpflichtung der Bewohner von Senioreneinrichtungen, das BVP-Gesprächsangebot auch in Anspruch zu nehmen

D eine professionelle Dokumentation sowie die Aktualisierung und Konkretisierung im Verlauf

E die Beachtung und Befolgung durch Dritte

Welche Aussage hinsichtlich des Rechts auf Selbstbestimmung trifft nicht zu?

A Das Recht auf Selbstbestimmung ist verfassungsrechtlich geschützt.

B Das Recht auf Selbstbestimmung ermöglicht dem Patienten, nicht gewollte Behandlung abzulehnen, auch wenn diese aus ärztlicher Sicht angezeigt ist.

C Das Recht auf Selbstbestimmung ist an die aktuelle Einwilligungsfähigkeit des Patienten zum Zeitpunkt der infrage stehenden Behandlung gebunden.

D Aus dem Prinzip des Respekts vor der Autonomie des Patienten folgt das Recht auf Selbstbestimmung.

E Das Recht auf Selbstbestimmung beinhaltet keinen Anspruch des Patienten auf jede gewünschte Behandlung. 


\section{7}

A Patientenverfügungen bieten die Möglichkeit, eigene Behandlungswünsche im Vorfeld einer schweren oder zum Tode führenden Krankheit festzulegen.

B In Notfallsituationen ist nichtärztliches Personal (z.B. Fachkräfte der Seniorenpflege oder des Rettungsdienstes) an Patientenverfügungen grundsätzlich nicht gebunden.

c Mit einer Vorsorgevollmacht wird eine Vertrauensperson ermächtigt, für den Fall der Entscheidungsunfähigkeit des Vollmachtgebers stellvertretend in eine Behandlung einzuwilligen oder diese abzulehnen.

D Der Bevollmächtigte ist an den erklärten oder mutmaßlichen Willen des Patienten gebunden.

E Patientenverfügung und Vorsorgevollmacht bieten eine Möglichkeit, das Recht auf Selbstbestimmung vorsorglich wahrzunehmen.

8 Eine der folgenden Maßnahmen gehört nicht zu den Inhalten von Behandlung im Voraus planen (BVP). Welche?

A die Ermittlung allgemeiner Einstellungen zum Leben und zum Einsatz medizinischer Maßnahmen bei schwerer Erkrankung

B die Klärung möglicher Therapie- und Behandlungsziele bei schwerer Erkrankung

C die Klärung und Dokumentation von Behandlungspräferenzen für mögliche zukünftige medizinische Entscheidungssituationen bei akuten medizinischen Krisen mit plötzlichem Verlust der Einwilligungsfähigkeit, bei akuter schwerer Erkrankung mit anhaltender Nichteinwilligungsfähigkeit und bei chronischen Erkrankungen mit dauerhafter Nichteinwilligungsfähigkeit

D die Ermittlung und Benennung einer Vertrauensperson, die als Stellvertreter für zukünftige medizinische Entscheidungen bevollmächtigt wird

E die vorausschauende Behandlungsplanung der onkologischen Zweit- und Drittlinientherapie bei fortgeschrittenen Krebserkrankungen
9 Welche Aussage zur Vorausplanung für akute Notfall- und Krisensituationen nach einer Beratung durch einen befähigten Gesprächsbegleiter und der Unterzeichnung aller Beteiligten trifft nicht zu?

A Rechtlich ist die Vorausplanung nicht bindend, da der Patient sich auch hätte kurzfristig anders entscheiden können.

B Um wirksam werden zu können, müssen Vorausverfügungen im Ernstfall vorliegen und auf Anhieb klar ersichtliche Handlungsanweisungen beinhalten.

C Für ärztliches und nichtärztliches Personal ist die Notfallplanung grundsätzlich auch in einer akuten Notfallsituation rechtlich bindend.

D Rettungsdienstmitarbeiter, die entgegen den in einem Notfallbogen niedergelegten Patientenwillen eine Reanimation beginnen, machen sich prinzipiell der strafbaren Körperverletzung schuldig.

E Die Idee eines Formulars, das eine ärztliche Anordnung über lebensverlängernde Maßnahmen mit der Unterschrift des Patienten (bzw. Vertreters) im Sinne einer Vorausverfügung verbindet, wurde in den 1990er-Jahren in den USA entwickelt.

Die Implementierung von Programmen zu Behandlung im Voraus planen (BVP) in der Region durch Vernetzung und Change Management beinhaltet einen der folgenden Faktoren nicht. Welchen?

A ein systematisches Konzept für die regionale Umstrukturierung eines Gesundheitswesens mit dem Ziel, einer patientenzentrierten Versorgung stärkeres Gewicht zu verleihen

B insbesondere Vorteile für Behandlungen bei aktueller oder dauerhafter Nichteinwilligungsfähigkeit von Patienten

C zunächst die Zusammenarbeit mit Institutionen und Personen, die von der Notwendigkeit und Sinnhaftigkeit des Systemwandels überzeugt sind

D als obligaten Bestandteil die Kooperation mit palliativmedizinisch qualifizierten und tätigen Netzwerken

E eine entsprechende Qualifizierung der BVP-Gesprächsbegleiter, regional einheitliche Formulare sowie die Definition und Einhaltung von Standards

\section{Ergänzendes Material}

Den Anhang finden Sie im Internet unter http://dx.doi.org/10.1055/s-0042-110711

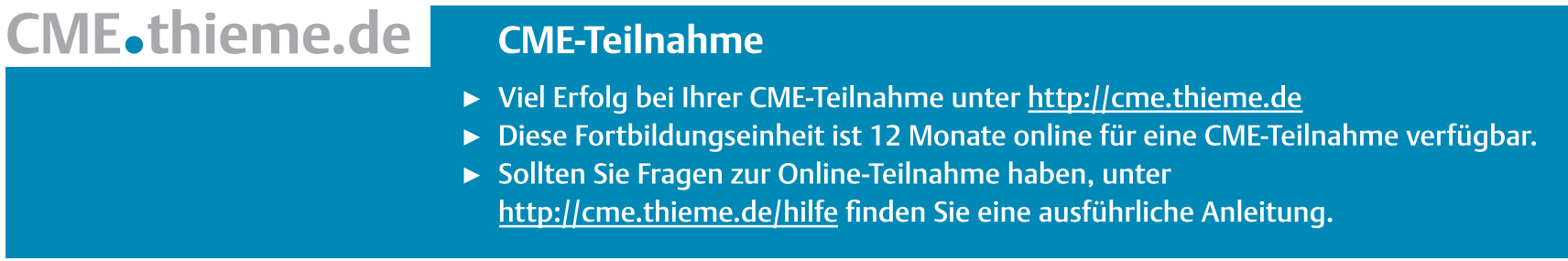

